\title{
10 Zusammenfassung
}

Ziel der vorliegenden Studie war es, kolonial motivierte Straßennamen in der deutschen Metropole zwischen 1884 und 1945 flächendeckend zu erheben und unter strukturell-onomastischen und diskursfunktionalen Fragestellungen umfassend $\mathrm{zu}$ analysieren. Dieses kolonialtoponomastische Forschungsanliegen reagiert auf den von der Koloniallinguistik eingehend beschriebenen Sachverhalt, dass der öffentliche Sprachgebrauch des späten 19. und frühen 20. Jahrhunderts im deutschsprachigen Raum in Bezug auf Kolonialismus und koloniale Themen bisher nur unzureichend erforscht ist. Vor diesem Hintergrund sollte am sprachlichen Alltagsgegenstand der Straßennamen empirisch, strukturell und diskursiv herausgearbeitet werden, wie und mit welchen sprachlichen Strukturen im Deutschen Reich eine spezifisch koloniale Belegung des (Alltags-)Raumes und der (Alltags-)Kommunikation evoziert werden sollte, die hinsichtlich der damit reproduzierten Gewissheiten untersucht wurde. Das Forschungsthema bezieht damit grundlegende sprachwissenschaftliche Fragestellungen auf den kolonialtoponomastischen Gegenstand:

Wer sagt was mit welchen Mitteln? Welche Sprache bildet kolonisatorische Identität im Alltag faktisch aus durch bloße Existenz von Kommunikation? Es ist die äußerliche Präsenz von Sprache als bloße Positivität eines Diskurses [...], die interessiert. (Warnke 2009: 33)

Das „Wer“ dieser Studie stellen die zeitgenössischen administrativen Namengeber dar, die „mit Mitteln“, nämlich mittels Benennungspraktiken kolonial motivierter Straßennamen über deren primäre Orientierungsfunktion hinaus „,kolonisatorische Identität im Alltag“ der deutschen Gesellschaft versprachlichten und im öffentlichen Raum fixierten.

Die Untersuchung dieses kolonialen Namenprojekts in der deutschen Metropole erfolgte in der Arbeit erstmalig in einem ortsübergreifenden Zugriff auf der Grundlage eines kriterienbasiert aus Groß- und Mittelstädten bestehenden historischen Ortskorpus. Auf dieser Basis wurde im Anschluss an etablierte Verfahren der empirischen Sprachwissenschaft ein systematisches Nameninventar aus der Perspektive ex ante identifiziert, inventarisiert und kategorisiert. Dabei konnte nachgewiesen werden, dass kolonial motivierte Straßenbenennungen strukturell beschreibbare und ortsübergreifend nachweisbare usuelle sprachliche Praxis darstellen. Die Analyse der nach linguistischen Kategorien annotierten über 520 historischen Datensätze zeigt die sprachliche Serialität der Straßennamen hinsichtlich ihrer onomastischen Strukturen, ihrer zeitlichen und räumlichen Verteilung und der mit ihnen verbundenen diskursiven Argumentationszusammenhänge, die das Potential zur Ableitung übergeordneter ๖ Open Access. ( 2021 Verena Ebert, publiziert von De Gruyter. (cc)BY-ND Dieses Werk ist lizenziert unter der Creative Commons Attribution-NoDerivatives 4.0 International Lizenz.

https://doi.org/10.1515/9783110718133-011 
Aussagen über die sprachlich vermittelten kolonisatorischen Wissenskonzepte klar erkennbar machen.

In der Einleitung zu dieser Arbeit wurden konkrete Untersuchungsfragen gestellt, die nun vor dem Hintergrund der Analyseergebnisse beantwortet werden sollen.

(1) In welchen Orten und Ortstypen des Deutschen Reichs sind kolonial motivierte Straßenbenennungen, die zwischen 1884 und 1945 von den städtischen Administrationen verfügt wurden, zu verzeichnen?

(2) In welchen gesellschaftspolitischen Zeiträumen (Kaiserreich, Weimarer Republik, Nationalsozialismus) sind kolonial motivierte Straßenbenennungen maßgeblich zu verzeichnen?

Hinsichtlich solcher raumzeitlichen Aspekte sind folgende Ergebnisse festzuhalten: Die hohe Zahl an nahezu 100 (99) Städten, in denen kolonial motivierte SN-Token erhoben werden konnten, verweist auf die große Relevanz, die der Etablierung kolonialdiskursiver Gewissheiten von Seiten der zeitgenössischen Administration für eine vergleichbare Anzahl an Groß- und Mittelstädten beigemessen wurde. Für den gesamten Untersuchungszeitraum ist die Entwicklung eines kolonial geprägten sprachlichen Usus mittels entsprechender Namenvergabepraktiken erst im diachronen Verlauf zu erkennen: Die ortsübergreifend-nationalen Formen kolonialer Place-Making-Prozesse sind weniger als ein Phänomen der faktischen Kolonialherrschaft in der Zeit des Kaiserreichs, sondern vielmehr als hochfrequente Prozesse für die Zeit nach dem faktischen Ende deutscher Kolonialherrschaft zu beschreiben. Der Kolonialmetropole Berlin (vgl. Heyden und Zeller 2002) ist hinsichtlich der Anzahl an entsprechenden Straßennamenvergabepraktiken bis 1919 eine Vorreiterrolle zuzusprechen, es werden aber auch bereits in weiteren Städten (Bremen, Chemnitz, Cuxhaven, Dresden, Düsseldorf, Erfurt, Hannover, Köln, Leipzig, Magdeburg, Weimar) kolonial motivierte Benennungsprozesse offensichtlich. Zwischen 1919 und 1945, insbesondere seit der nationalsozialistischen Machtübernahme 1933, können umfangreiche ortsübergreifend-nationale Fixierungsprozesse kolonialer Kommemorationen in den öffentlichen Raum des Deutschen Reichs mittels Straßennamenverfügungen festgestellt werden, mit denen kolonisatorische Gewissheiten sprachlich vermittelt werden sollten. Dabei waren offenbar zwei äußere Ereignisse maßgeblich: Die Politik der nationalsozialistischen Gleichschaltung der Kolonialbewegung bis auf die kommunalen Ebenen stellt insofern einen auch sprachlichen Wendepunkt dar, als dass der Reichskolonialbund mit seinen lokalen Kreisverbänden solche Namenverfügungspraktiken bei den nationalsozialsozialistisch-städtischen Administrationen vorantreiben konnte. Dar- 
über hinaus wurden koloniale Straßenbenennungen (neben weiteren) spätestens durch den „Runderlaß des Reichsministers des Innern“ (15.07.1939) von staatlicher Seite unterstützt. Gerade die Erhebung kolonial motivierter Straßenbenennungen für einzelne Städte der annektierten Gebiete am Ende der 1930er und Anfang der 1940er Jahre ist ein Hinweis darauf, dass sie im NS-System (neben weiteren) einen Themenbereich darstellten, die innerhalb zeitgenössischer narrativer Muster des nationalsozialistischen Deutschen Reichs als unmittelbar relevant angesehen wurden.

(3) Welche musterhaften sprachstrukturellen und diskursfunktionalen Eigenschaften weisen die Konstruktionen im inventarbezogenen Zugriff auf?

In dieser Arbeit konnte gezeigt werden, dass innerhalb des kolonialen Namenprojekts in der Metropole prototypische sprachstrukturelle Konstruktionen Verwendung fanden, denen musterhafte Diskursfunktionen hinsichtlich der damit versprachlichten kolonisatorischen Gewissheiten zugeschrieben werden können. Dies gilt schon für den Bestand der Modifikator-Konstruktionen an sich: Etwa $84 \%$ aller erhobenen über 520 SN-Token beschränken sich auf gerade einmal 27 MOD-Types, die mit usuellen Klassifikatoren verfügt wurden. Das hohe Maß an Systematizität ist auch im Hinblick auf die lexikologisch-onymische Kategorisierung der über 520 Modifikator-Konstruktionen festzustellen: Etwa 59 \% bzw. 36 \% der Konstruktionen weisen anthroponymische bzw. toponymische ModifikatorKonstruktionen auf, für die (durch die Auswertung zeitgenössischer Zuschreibungen) musterhafte Kommemorationen herauszustellen sind, mit denen personalisierte und lokalisierte Wissenskonzepte in Bezug auf die deutschen Kolonialakteure und die von Seiten der deutschen Kolonialmacht kolonisierten Räume als eindeutige Machthierarchien im deutschsprachigen (Alltags-)Raum fixiert werden sollten. Das Beispiel der kolonialen Straßennamen belegt damit die vor einigen Jahren formulierte These von Warnke und Stolz (2013: 483) deutlich: „Im kolonialen Dispositiv ist der personale, lokale und temporale Verweisraum also nicht nur binär strukturiert, sondern stets auch klar bewertet. Die kolonisatorische Origo ist immer eine der überlegenen Position“. Die sprachlich vermittelte eurozentristische Haltung konnte für die innerhalb des zweitgrößten Teilbestandes detoponymischer Konstruktionen festzustellenden deoikonymischen Modifikatoren herausgearbeitet werden: Sie verweisen nicht auf beliebige Orte in den kolonisierten Räumen, sondern ausschließlich auf administrative Hauptorte und/oder handelsspezifische Zentren der deutschen Kolonialmacht mit quantitativ dominanten georeferenzierenden Bezügen zu Deutsch-Südwestafrika als zeitgenössisch einziger Siedlungskolonie. 
Damit kann das koloniale Namenprojekt, auch und gerade nach 1919, weniger als eine „Vermarktung des Fremden“ (Wolter 2005) herausgestellt werden. In plakativer Zuspitzung lässt sich damit sagen: Während in unterschiedlichen zeitgenössischen Textsorten nicht selten Koloniales in stereotypischen Themenfeldern von exotisch-imaginierter Ferne und Fremde in Übersee (vgl. Warnke 2009: 50-51) verhandelt wird, handelt es sich bei dem Alltagsgegenstand kolonial motivierter Straßennamen vielmehr um den Versuch einer Reinstallierung kolonialer Erfahrung (Abb. 1, Kap. 2.2.4) im Sinne kolonisatorischer Superiorität. Dass die kommunikative Handlungsmacht ausschließlich für die Seite der Kolonisatoren auszumachen ist, wird gerade durch die weitgehend erst in nationalsozialistischer Zeit verfügten Konstruktionen deutlich, mit denen aus zeitgenössischer Sicht die erfolgreich geführten Kolonialkriege und die Kolonialhelden geehrt und/oder gewürdigt werden sollten. Die nationalsozialistische Kolonialbewegung schaffte sich durch die Anregung ortsübergreifender Namenvergabeprozesse auch über die Fortführung älterer Namenmuster hinaus eigene Kommemorationen, die sich durch partiell übereinstimmende, aber auch davon abweichende Konstruktionsmuster gegenüber der faktischen Kolonialzeit auszeichnen. In diesem Zusammenhang ist auch die ortsübergreifend-nationale Ehrung von Carl Peters mit einer hochfrequenten Zahl an Straßennamen nach 1933 entgegen seiner noch in der Kaiserzeit erfolgten Dienstentlassung und entsprechender Geringschätzung einzuordnen.

(4) Welche musterhaften Formen kolonialer Raumreferenzierung und -belegung sind im inventarbezogenen Zugriff verzeichnen?

Die vorliegende Studie konnte die gebündelte Verfügung von SN-Token in der Form von Kolonialclustern als einen raumlinguistischen Prototyp aufzeigen. Auch für die innerhalb solcher Cluster zu verortenden Namen konnten ortsübergreifende sprachstrukturelle und diskursfunktionale Muster aufgezeigt werden, mit denen personenbezogene oder personenbezogen-lokalisierte Kommemorationen in Bezug auf Kolonialismus und koloniale Themen hervorgebracht wurden. Anhand der durchschnittlich größeren Anzahl an innerhalb von Clustern verfügten kolonial motivierten SN-Token in Großstädten, die mit der Anlegung größerer Siedlungen und/oder Viertel $\mathrm{zu}$ begründen ist, konnte eine Attraktivitätsskala kolonialer Kommemorationen herausgearbeitet werden. Eine besondere Attraktivität geht demnach von sprachlichen Strukturen aus, mit denen gebündelte kolonisatorische Wissenskonzepte zu Kolonialakteuren oder den kolonisierten Räumen und die maßgeblich daran beteiligten Kolonialakteure evoziert werden sollten. Neben den usuellen Konstruktionsmustern sind 
innerhalb solcher Cluster auch singuläre Phänomene mit teilweise über die kolonialen Motiviken hinausgehenden Zuschreibungen festzustellen.

(5) Welche zeitgenössische Relevanz ist kolonial motivierten Straßenbenennungen im inventarbezogenen Zugriff hinsichtlich ihres Benennungsstatus als Neu- oder Umbenennungen zuzuschreiben?

Die Untersuchung der historisch-synchronen Relevanz der Namenvergabepraktiken vor dem Hintergrund ihres Benennungsstatus hat ergeben, dass koloniale Straßenbenennungen (mit weiteren) vorrangig im Zusammenhang der Erschließung städtischer Peripheriegebiete $\mathrm{zu}$ verorten sind. Die gebündelte Vergabepraxis kolonial motivierter Straßennamen stellte einen von mehreren etwaigen Themenbereichen vor dem Hintergrund des zeitgenössischen Benennungsbedarfs neu angelegter Siedlungen oder Viertel in den Randgebieten der Städte dar, für den sich besonders die Vereine der Kolonialbewegung des Deutschen Reichs einsetzten. Koloniale Benennungen, die im Zuge der Tilgung älteren Namenmaterials verfügt wurden, stellen ein untergeordnetes Phänomen dar und sind maßgeblich für deanthroponymische Konstruktionen festzustellen, die als Einzelbenennungen verfügt wurden. Wenngleich koloniale Umbenennungsprodukte mehrheitlich auf lokal-parteiliche Intentionen der nationalsozialistischen Stadtverwaltungen zurückzuführen sind, sind sie nicht mit den Umbenennungsaktionen seit 1933 (Adolf-Hitler-Straße u. dgl.) in den mittelalterlichen Stadtkernen vergleichbar.

(6) Welche Argumentationsstrukturen manifestieren sich in expliziten Aussagenzusammenhängen, die kolonial motivierte Straßenbenennungen propositional einbetten und begründen?

Im Gegensatz zu den inventarbezogen-toponomastischen Analysen konnten argumentatorisch-diskursbezogene Untersuchungen zeitgenössischer expliziter Aussagenzusammenhänge nur einzelorts- bzw. einzelfallbezogen innerhalb der jeweiligen Teilkapitel erfolgen. Das liegt daran, dass die Quellenlage der hierfür erforderlichen Benennungsakten unterschiedlich dicht ausfällt. Umso wertvoller sind die mit guter Quellenlage einhergehenden einzelortsbezogenen Befunde aus administrativen Benennungsakten und Benennungsmotiviken in historischen Straßenverzeichnissen, die für die städtische Öffentlichkeit angelegt wurden. Durch die Berücksichtigung und Auswertung von Zuschreibungen, die innerhalb solcher metasprachlichen Äußerungen vorgenommen werden, können die mit kolonialen Namenvergabepraktiken intendierten epistemischen Lesarten von Seiten der Namengeber aufgedeckt werden. Unabhängig von ihrem Benennungsstatus als Erst- bzw. Umbenennungen sind dabei für die nach 1919 verfügten Inventare kolonialrevisionistische Argumenta- 
tionstopoi festzustellen, die in den jeweiligen Texten explizit und implizit verhandelt werden. Die kolonialrevisionistischen Argumentationsmuster der Rückforderung entsprechen dabei in auffälliger Weise den Argumentationen zeitgenössischer Reichstagsprotokolle der 1920er Jahre, die bspw. Kämper (2016) hinsichtlich argumentativ-lexikalischer Gesichtspunkte ausgewertet hat.

Die Ergebnisse der hier vorliegenden Studie zu kolonial motivierten Straßennamen belegen die Relevanz einer sprachwissenschaftlichen Erforschung sekundärer Straßennamen in strukturell-onomastischer und diskursfunktionaler Perspektive. Es konnte nachgewiesen werden, dass hinter den praktischen Aspekten kolonial motivierter Straßenbenennungen ortsübergreifend-nationale sprachliche Interessen rekonstruiert werden können, anhand derer eine nationale Symbolik (vgl. Jessen 2006: 3) offensichtlich wird. Diese Arbeit stellt damit zum einen ein Plädoyer für den Ausbau der Studien zu sekundären Straßennamen in der Onomastik und zum anderen ein Plädoyer für die stärkere Berücksichtigung der Onyme im Bereich der sogenannten kolonialen Metropolen in der Kolonialtoponomastik dar. Erforderlich ist dazu eine ortsübergreifende Erhebung des Namenmaterials unter genauer Aufarbeitung der historischen Vergabeprozesse, seine Inventarisierung und Kategorisierung mit etablierten Methoden der empirischen Sprachwissenschaft und seine intensive Analyse, die mit der Erhebung akteursgebundener Intentionen der administrativen Namengeber und den jeweiligen Argumentationsstrukturen auch sprachlich-diskursive Anteile umfasst. Im Hinblick auf die von Kämper et al. (2016: 5) aufgezeigte Aufgabe sprachwissenschaftlicher Teildisziplinen, „die Multidisziplinarität der Diskursanalyse disziplinär zu ordnen“, ist unter jenen Voraussetzungen auch zu fragen, inwieweit sekundäre Straßennamen hinsichtlich ihrer epistemischen Lesart nicht nur als theoretischer Bestandteil der linguistischen Diskursanalyse zu verhandeln, sondern auch in der Praxis entsprechend zu untersuchen sind.

Das innerhalb dieser Arbeit vollständig quellenbasiert erhobene Inventar von über 520 kolonial motivierten Straßenbenennungen bildet den für 1945 ermittelten Ist-Zustand kolonialer Straßennamen. Es stellt zugleich eine ideale Datengrundlage für weitere sprachwissenschaftliche Fragestellungen nach 1945 dar, „denn nur auf Grundlage eines vergleichbar ermittelten Datensets lassen sich [...] überhaupt erst Weiterungen der Namengeschichte angemessen verfolgen und einordnen“ (Stolz und Warnke 2017: 211). Punktuelle Stichproben zeigen den Ausbau der Kolonialcluster in den städtischen Peripheriebereichen nach 1945, der zu themenkohärenten Weiterbenennungen der in nächster Nähe gelegenen Straßenzüge führte, bspw. in Duisburg-Huckingen (u. a. Daressalamstraße, Lomestraße, Sansibarstraße, Togostraße), Hannover-Badenstedt (u. a. Togoweg, Windhukstraße) und Wuppertal-Barmen (Togostraße). Auch Tilgungs- 
praktiken einzelner deanthroponymischer Konstruktionen, für die in sprachhistorischer Perspektive eindeutige koloniale Kommemorationen nachzuweisen sind, können ausgehend von dem in dieser Arbeit zusammengestellten und analysierten Inventar in den Blick genommen werden. Zum einen ergeben sich Unterschiede der sprachlichen Praktiken nach 1945 in den westlichen Besatzungszonen auf der einen und in der SBZ auf der anderen Seite (vgl. Schulz und Ebert 2016: 362). Hier erscheint eine intensive Analyse auf der Basis des in dieser Studie erhobenen Materials vielversprechend. Zum anderen sind seit etwa 1990 charakteristische Umbenennungen, etwa für Köln-Nippes (Carl-Peters-Straße, Lüderitzstraße), München-Trudering (Von-Trotha-Straße) oder Hannover-Badenstedt (Lettow-Vorbeck-Allee) festzustellen. Toponomastische Fragen hinsichtlich der lexikologischonymischen Zuordnung der jeweiligen Modifikatoren sind hier im Kontrast zu den Benennungen auch an die Umbenennungsprodukte zu richten. Von besonderem Interesse wird dabei eine Analyse postkolonialer Zuschreibungen sein.

Der in dieser Studie erreichte Beschreibungsstand könnte damit den Ausgangspunkt für anschließende Untersuchungen bilden, die Analyseperspektiven $\mathrm{zu}$ sprachlichen Prozessen der Tilgung und Umbenennung insbesondere seit 1945 bilden. Für sie wäre zu fragen, welche kommunikativ-sprachliche Dimension mit (de-) kolonisatorischer Wissensproduktion einhergeht. Die ortsübergreifende Zusammenstellung solcher Tilgungs- und Umbenennungsprozesse erforderte wie in dieser Studie Analysen zu raumzeitlichen sowie strukturbezogen-onomastischen Aspekten und der sich daraus ergebenden diskursfunktionalen Eigenschaften. Neben den vorrangig toponomastischen Analysen könnten zudem für die jüngeren und vor allem aktuellen Umbenennungsdebatten um kolonial motivierte Straßennamen metasprachliche Daten zusammengestellt und erhoben werden, die sodann nach prototypischen Argumentationsmustern verschiedener Akteursgruppen ausgewertet werden könnten. Für solche dringend gebotenen Studien wäre die hier vorliegende Arbeit Vorarbeit und Ausgangspunkt zugleich. 
